\title{
SELEÇÃO DE CARACTERES CORRELACIONADOS EM CUBIU (Solanum sessiliflorum Dunal) EMPREGANDO A ANÁLISE DE TRILHA.
}

\author{
Danilo Fernandes da SILVA FILHO', Clodoaldo José da ANUNCIAÇÃo \\ FILHO $^{2}$, Hiroshi NODA ${ }^{1}$, Odemar Vicente dos REIS ${ }^{3}$
}

RESUMO - Vinte e nove populações naturais de cubiu (Solanum sessiliflorum Dunal) da coleção do Programa de Melhoramento Genético de Hortaliças do Instituto Nacional de Pesquisas da Amazônia-INPA, foram avaliadas na região Nordeste do Brasil. $\mathrm{O}$ experimento foi conduzido na Estação Experimental da Empresa Pernambucana de Pesquisa Agropecuária-IPA, em Vitória de Santo Antão, Estado de Pernambuco. Adotou-se o delineamento experimental em blocos casualizados com 4 repetições. A unidade experimental foi constituida de uma área de $7,5 \mathrm{~m}^{2}$ contendo 5 plantas úteis Coletaram-se dados referentes ao diâmetro do caule (DC), altura da planta (AP), área da folha (AF), largura do fruto (LF), comprimento do fruto (CF), número de lóculos (NL), espessura da polpa (EP),número total médio de frutos por planta (NMF), peso médio dos frutos (PMF), peso total de frutos (PEF) e teor de sólidos solúveis totais (BRIX). Empregou-se a Ánalise de Trilha para desdobrar os coeficientes de correlação genotípica, e observar a inlfuência dos efeitos destes caracteres, na produção de frutos de cubiu. Verificou-se que o maior número de associações positiva e significativa entre os pares de caracteres avaliados ocorreu com a espessura da polpa (EP). A Análise de Trilha desenvolvida sobre os dez caracteres, mostrou que o número total médio de frutos por planta (NMF) e a espessura da polpa (EP) foram os caracteres que mais influenciaram direta e positivamente sobre a produção estimada de frutos de cubiu.

Palavras-chave: Solanum sessiliflorum, Solanaceae, associação entre caracteres, melhoramento genético, análise de trilha.

Selection of Correlated Characters in Cubiu (Solanum sessiliflorum Dunal) Using Path Coefficient Analysis.

ABSTRACT - Twenty nine accessions of orinoco apple ( $S$. sessiliflorum) from the germplasm collection of the Vegetable Breeding Program of the National Research Institute of Amazonia (INPA) were evaluated in Northeastern Brazil. The trial was carried out in the Vitória de Santo Antão Experimental Station, of the Pernambuco State Research Enterprise (IPA), Pernambuco. A randomized complete block design, with four replications, was used. The following characters of agronomic importance were evaluated: stem diameter; plant height; leaf area; fruit width; fruit length; locule number; pulp thickness; total fruit number per plant; average fruit weight; total fruit yield; and total soluble solids (Brix). The genotypic correlations among characters were estimated and used in path coefficient analysis to determine their influence on yield. The greatest number of positive and significant correlations occurred with pulp thickness. Total fruit number per plant and pulp thickness were the characters with the greatest direct and positive influence on yield of cubiu.

Key-words: (Solanum sessiliflorum), Solanaceae, association among characters, plant breeding, path coefficient analysis.

\section{INTRODUÇÃO}

O cubiu (S. sessiliflorum) também conhecido como topiro, tupiro, cocona, orinoco apple e peach tomato, é uma espécie nativa da Amazônia amplamente distribuída na região equatorial úmida brasileira, peruana e colombiana.

INPA / CPCA - C.P. 478, 69.011-970, Manaus, Amazonas, Brasil.

2 Universidade Federal Rural de Pernambuco, Departamento de Fitotecnia, 51.171-030, Recife, Pernambuco, Brasil.

3 Empresa Pernambucana de Pesquisa Agropecuária, C.P. 1022, 50.000-000, Recife, Pemambuco, Brasil. 
De maneira geral, esta Solanaceae arbustiva vem sendo mantida nos sítios dos pequenos produtores rurais onde é cultivada em pequena escala. Os frutos do cubiu são ricos em ferro, niacina (Vit. $\mathrm{B}_{5}$ ) ácido cítrico e pectina (INN, 1977; PAHLEN, 1977; SILVA FILHO, 1994).

Por essa razão, são utilizados como alimento e medicamento pelas familias tradicionais da Amazônia. Como alimento são consumidos in natura ou nas formas de sucos, doces, compotas, geléias e tempero de sopas de peixe. Como medicamento, no controle de coceiras da pele e para reduzir os niveis elevados de colesterol, glicose e ácido úrico no sangue.

A correlação entre caracteres é útil na determinação dos componentes de um caráter complexo, como a produção. Entretanto, ela não dá de maneira exata, a importância relativa das influências diretas e indiretas de cada um desses caracteres (VENCOVSCKY e BARRIGA, 1992). Dada a importância das relações causais entre caracteres nas estratégias de melhoramento, WRIGHT (1934) propôs o método da análise de trilha (path coefficient analysis) o qual permite desdobrar o coeficiente de correlação em componentes de efeitos diretos e indiretos, possibilitando melhor entendimento de todos os fatores básicos (causas) e suas variáveis resultantes (efeitos).

Com o surgimento de programas específicos para uso em microcomputadores, a análise de trilha tem sido muito utilizada no melhoramento das mais variadas espécies. DEWEI e LU (1959) e BHATT (1970) desenvolveram pesquisas com diversos genótipos de trigo.
LENKA E MISRA (1973), SHRIVASTAVA E SHARMA (1976) e RANGEL (1979) utilizaram este método no melhoramento do arroz. DUARTE e ADAMS (1972) e OLIVEIRA et al. (1990) empregaram a técnica no estudo da variabilidade genética em cultivares e progênies de feijão. No melhoramento de clones de seringueira, PAIVA et al. (1982) e VASCONCELOS e ABREU (1983) usaram a mesma metodologia obtendo resultados compensadores.

Este trabalho tem o objetivo de estimar a magnitude da influência de caracteres associados na produção de cubiu.

\section{MATERIAL E MÉTODOS}

Vinte e nove populações naturais de cubiu originárias de diversas partes da Amazônia brasileira, peruana e colombiana foram avaliadas (Fig. 1).

$O$ experimento foi conduzido na Estação Experimental da Empresa Pernambucana de Pesquisa AgropecuáriaIPA, a 1,5 km de Vitória de Santo Antão, Estado de Pernambuco, situada nas coordenadas geográficas, segundo a CONDEP/IPA (1987): Latitude $08^{\prime \prime} 08^{\prime \prime} 00^{\circ} \mathrm{S}$, Longitude $35^{\circ} 00^{\prime \prime} 00^{\prime} \mathrm{W}$ e a $146 \mathrm{~m}$ ao nivel do mar.

O clima local é classificado como $\mathrm{C}_{1} \mathrm{~S}_{2} \mathrm{~A}$ 'a seco sub-úmido megatérmico, no esquema de Tornthweith. O equilibrio hídrico, quando não há excesso nem deficiência, é atingido entre os messes de abril e julho. Do mês de setembro até março vão se acumulando deficiências hídricas que apresentam valores críticos nos meses de novembro e dezembro (ENCARNAÇÃO, 1980).

$\mathrm{O}$ solo e o relevo predominantes 


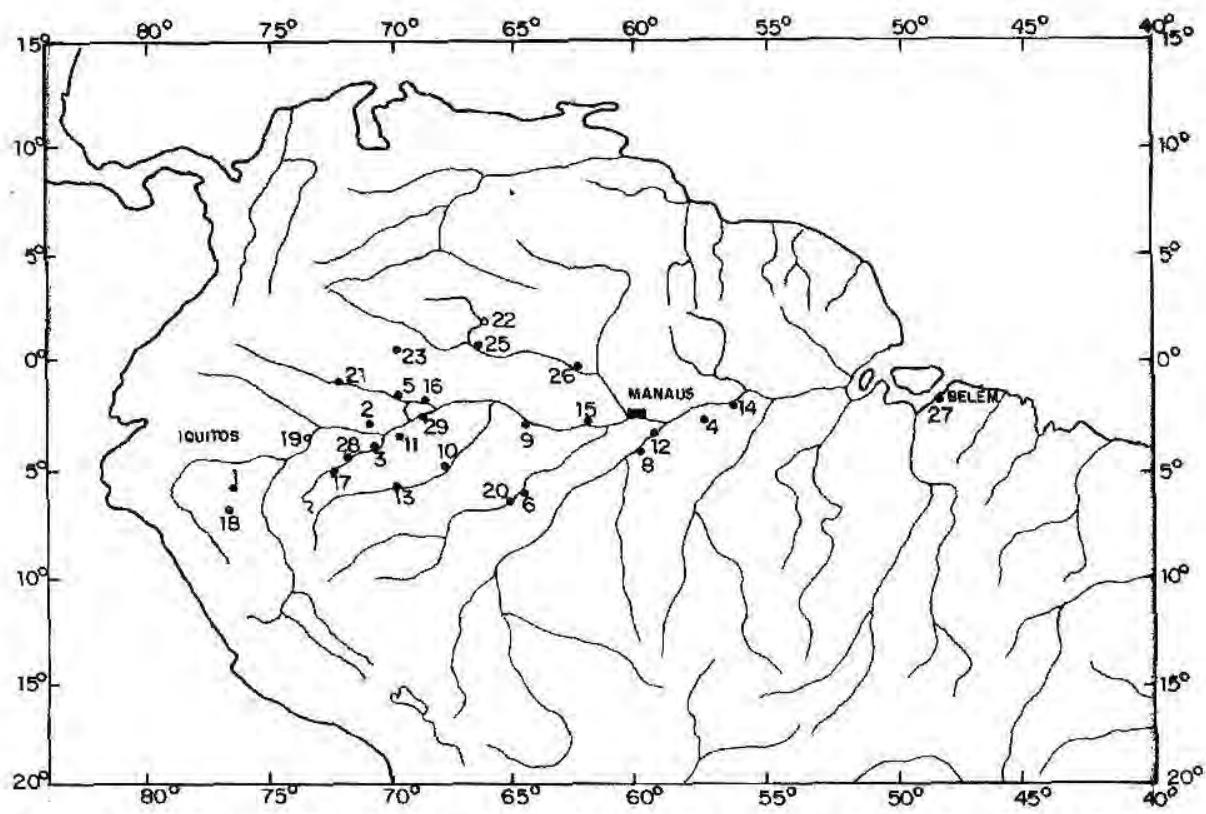

Figura 1. Origem geográfica de 29 populações de cubiu (S. sessilifforum) avaliadas na Zona da Mata do Estado de Pernambuco em 1992/1993. 1.Yurimaguas; 2. Arara; 3. Atalảia do Norte; 4. Ponta Alegre; 5. Ipiranga; 6. Canutama; 7. Umariaçú; 8. Borba; 9. Tefé; 10. Carauari; 11. Benjamin Constant; 12. Nova Olinda; 13. Eirunepé; 14. Parintins; 15. Coari; 16. Betânia; 17. Estirão do Equador; 18. Tarapoto; 19. Iquitos; 20. Lábrea; 21. Putumayo; 22. Cucui; 23. Vila Bitencourt; 24. Palmeiras; 25. São Gabriel da Cachoeira; 26. Barcelos; 27. Belém; 28. Mayuruna; e 29. São Paulo de Olivença.

na região são formados por uma associação de : latossolo vermelhoamarelo distrófico com A moderado, textura argilosa; podzólico vermelhoamarelo orto, relevo ondulado; podzólico vermelho-amarelo latossólico, textura argilosa, relevo forte ondulado e montanhoso (CONDEPE/IPA, 1987).

A semeadura foi feita em bandejas de isopor no dia 11de julho de 1992. Utilizou-se como substrato um solo autoclavado a $120{ }^{\circ} \mathrm{C}$ por duas horas. As bandejas foram colocadas em casa de vegetação.

No $30^{\circ}$ dia após a semeadura, foi efetuada a repicagem das plântulas para bandejas com celas mais espaçosas. Colocou-se somente uma plântula por cela.
O preparo da área foi realizado no fim do mês de agosto de 1992, e constou de uma aração e uma gradagem.

A adubação na cova consistiu de $70 \mathrm{~g}$ de superfosfato triplo, $50 \mathrm{~g}$ de cloreto de potássio e $20 \mathrm{~g}$ de sulfato de amônio.

O plantio foi realizado no dia $11 \mathrm{de}$ setembro de 1992. Aos quinze dias após o transplante, foi feita uma adubação em cobertura com $10 \mathrm{~g}$ de uréia/planta, repetindo-se mensalmente até o dia $11 \mathrm{de}$ janeiro de 1993.

As avaliações dos caracteres estudados foram feitas com base em cinco plantas úteis de cada parcela. Os dados foram anotados individualmente e depois calculadas as médias aritméticas.

Considerou-se de importância 
agronômica os caracteres diâmetro do caule (DC) a três centímetros do nível do solo, altura da planta (AP), área da folha (AF), largura do fruto (LF), comprimento do fruto $(\mathrm{CF})$, número de lóculos (NL), espessura da polpa (EP), número total médio de frutos por planta (NMF), peso médio dos frutos (PMF), produção estimada de frutos (PEF) até o término do ciclo vital da planta, e teor de sólidos solúveis totais (BRIX).

Adotou-se o delineamento experimental em blocos casualizados com quatro repetições. A unidade experimental consistiu de uma parcela de $7,5 \mathrm{~m}^{2}$ contendo 5 plantas úteis num espaçamento de $1,00 \mathrm{~m}$ entre plantas e $1,50 \mathrm{~m}$ entre as fileiras. microcomputador utilizando os recursos do programa GENES desenvolvido por CRUZ (1990). Na Fig. 2, são apresentadas através de um diagrama, as interrelações das variáveis envolvidas na análise de trilha.

Os coeficientes de caminhamento foram obtidos pela solução dos sistemas de equações que expressam o relacionamento básico entre as correlações e seus componentes desdobrados em efeitos diretos e indiretos, seguindo os procedimentos adotados por alguns pesquisadores (DEWEI \& LI, 1959; LENKA \& MISRA, 1973; RANGEL, 1979 \& PAIVA et al., 1982). As equações estabelecidas encontram-se abaixo discriminadas:

\begin{tabular}{|l}
$r_{1 x}=P_{1 x}+r_{12} P_{2 x}+r_{13} P_{3 x}+r_{14} P_{4 x}+r_{15} P_{5 x}+r_{16} P_{6 x}+r_{17} P_{7 x}+r_{18} P_{8 x}+r_{19} P_{9 x}+r_{110} P_{10}$ \\
$r_{2 x}=r_{21} P_{1 x}+P_{2 x}+r_{23} P_{3 x}+r_{24} P_{4 x}+r_{25} P_{5 x}+r_{26} P_{6 x}+r_{27} P_{7 x}+r_{28} P_{8 x}+r_{29} P_{9 x}+r_{210} P_{10}$ \\
$r_{3 x}=r_{31} P_{1 x}+r_{32} P_{2 x}+P_{3 x}+r_{34} P_{4 x}+r_{35} P_{5 x}+r_{36} P_{6 x}+r_{37} P_{7 x}+r_{38} P_{8 x}+r_{39} P_{9 x}+r_{310} P_{10}$ \\
$r_{4 x}=r_{41} P_{1 x}+r_{42} P_{2 x}+r_{43} P_{3 x}+P_{4 x}+r_{45} P_{5 x}+r_{46} P_{6 x}+r_{47} P_{7 x}+r_{48} P_{8 x}+r_{49} P_{9 x}+r_{410} P_{10}$ \\
$r_{5 x}=r_{51} P_{1 x}+r_{52} P_{2 x}+r_{53} P_{3 x}+r_{54} P_{4 x}+P_{5 x}+r_{56} P_{6 x}+r_{57} P_{7 x}+r_{58} P_{8 x}+r_{59} P_{9 x}+r_{510} P_{10}$ \\
$r_{6 x}=r_{61} P_{1 x}+r_{62} P_{2 x}+r_{63} P_{3 x}+r_{64} P_{4 x}+r_{65} P_{5 x}+P_{6 x}+r_{67} P_{7 x}+r_{68} P_{8 x}+r_{69} P_{9 x}+r_{610} P_{10}$ \\
$r_{7 x}=r_{71} P_{1 x}+r_{72} P_{2 x}+r_{73} P_{3 x}+r_{74} P_{4 x}+r_{75} P_{5 x}+r_{76} P_{6 x}+P_{7 x}+r_{78} P_{8 x}+r_{79} P_{9 x}+r_{710} P_{10}$ \\
$r_{8 x}=r_{81} P_{1 x}+r_{82} P_{2 x}+r_{83} P_{3 x}+r_{84} P_{4 x}+r_{85} P_{5 x}+r_{86} P_{6 x}+r_{87} P_{7 x}+P_{8 x}+r_{89} P_{9 x}+r_{810} P_{10}$ \\
$r_{9 x}=r_{91} P_{1 x}+r_{92} P_{2 x}+r_{93} P_{3 x}+r_{94} P_{4 x}+r_{95} P_{5 x}+r_{96} P_{6 x}+r_{97} P_{7 x}+r_{98} P_{8 x}+P_{9 x}+r_{910} P_{10}$ \\
$r_{10 x}=r_{101} P_{1 x}+r_{102} P_{2 x}+r_{103} P_{103}+r_{104} P_{4 x}+r_{105} P_{5 x}+r_{106} P_{6 x}+r_{107} P_{7 x}+r_{108} P_{8 x}+r_{109} P_{9 x}+P_{10 x}$
\end{tabular}

As variáveis avaliadas foram utilizadas para estimar os coeficientes de correlação genética entre os onze caracteres estudados, para efetuar a análise de trilha e demonstrar o significado desta técnica em relação à influência que os caracteres DC, AP, AF, LF, CF, NL, EP, NMF, PMF e BRIX exercem sobre a produção estimada de frutos de cubiu (PEF). Os dados experimentais foram processados em
Nestas equações os r's correspondem as correlações genotípicas. Os P's medem os efeitos diretos; os outros termos, os efeitos indiretos de uma variável sobre a outra.

$\mathrm{Na}$ solução do sistema, foi empregado o cálculo matriclal (Matriz $10 \times 10$ ), encontrando-se os valores dos P's na matriz vetor.

$O$ efeito residual $P_{x 10}$ foi obtido através da equação: 
$1=\mathrm{P}^{2}+110 \mathrm{P}_{1 \mathrm{x}}^{2}+\mathrm{P}_{2 \mathrm{x}}^{2}+\mathrm{P}_{3 \mathrm{x}}^{2}+\mathrm{P}_{4 \mathrm{x}}^{2}+\mathrm{P}_{5 \mathrm{x}}^{2}+\mathrm{P}_{6 \mathrm{x}}^{2}+\mathrm{P}^{2}{ }_{7 \mathrm{x}}+\mathrm{P}_{8 \mathrm{x}}^{2}+\mathrm{P}_{9 \mathrm{x}}^{2}+\mathrm{P}_{10 \mathrm{x}}^{2}+2 \mathrm{P}_{1 \mathrm{x}}+\mathrm{r}_{12} \mathrm{P}_{2 \mathrm{x}}+2 \mathrm{P}_{1 \mathrm{x}}+\mathrm{r}_{13} \mathrm{P}_{3 \mathrm{x}}+2 \mathrm{P}_{1 \mathrm{x}}+$ $\mathrm{r}_{14} \mathrm{P}_{4 \mathrm{x}}+2 \mathrm{P}_{1 \mathrm{x}}+\mathrm{r}_{15} \mathrm{P}_{5 \mathrm{x}}+2 \mathrm{P}_{1 \mathrm{x}}+\mathrm{r}_{16} \mathrm{P}_{6 \mathrm{x}}+2 \mathrm{P}_{1 \mathrm{x}}+\mathrm{r}_{17} \mathrm{P}_{7 \mathrm{x}}+2 \mathrm{P}_{1 \mathrm{x}}+\mathrm{r}_{18} \mathrm{P}_{8 \mathrm{x}}+2 \mathrm{P}_{1 \mathrm{x}}+\mathrm{r}_{19} \mathrm{P}_{9 \mathrm{x}}+2 \mathrm{P}_{1 \mathrm{x}}+\mathrm{r}_{10} \mathrm{P}_{10 \mathrm{x}}+2 \mathrm{P}_{2 \mathrm{x}}+\mathrm{r}_{23} \mathrm{P}_{3 \mathrm{x}}+$ $2 \mathrm{P}_{2 \mathrm{x}}+\mathrm{r}_{24} \mathrm{P}_{4 \mathrm{x}}+2 \mathrm{P}_{2 \mathrm{x}}+\mathrm{r}_{25} \mathrm{P}_{5 \mathrm{x}}+2 \mathrm{P}_{2 \mathrm{x}}+\mathrm{r}_{26} \mathrm{P}_{6 \mathrm{x}}+2 \mathrm{P}_{2 \mathrm{x}}+\mathrm{r}_{27} \mathrm{P}_{7 \mathrm{x}}+2 \mathrm{P}_{2 \mathrm{x}}+\mathrm{r}_{28} \mathrm{P}_{8 \mathrm{x}}+2 \mathrm{P}_{2 \mathrm{x}}+\mathrm{r}_{29} \mathrm{P}_{9 \mathrm{x}}+2 \mathrm{P}_{2 \mathrm{x}}+\mathrm{r}_{210} \mathrm{P}_{10 \mathrm{x}}+2 \mathrm{P}_{3 \mathrm{x}}+$ $\mathrm{r}_{34} \mathrm{P}_{4 \mathrm{x}}+2 \mathrm{P}_{3 \mathrm{x}}+\mathrm{r}_{35} \mathrm{P}_{5 \mathrm{x}}+2 \mathrm{P}_{3 \mathrm{x}}+\mathrm{r}_{36} \mathrm{P}_{6 \mathrm{x}}+2 \mathrm{P}_{3 \mathrm{x}}+\mathrm{r}_{37} \mathrm{P}_{7 \mathrm{x}}+2 \mathrm{P}_{3 \mathrm{x}}+\mathrm{r}_{38} \mathrm{P}_{8 \mathrm{x}}+2 \mathrm{P}_{39} \mathrm{P}_{9 \mathrm{x}}+2 \mathrm{P}_{3 \mathrm{x}}+\mathrm{r}_{310} \mathrm{P}_{10}+2 \mathrm{P}_{4 \mathrm{x}} \mathrm{r}_{45} \mathrm{P}_{5 \mathrm{x}}+$ $2 \mathrm{P}_{4 x} \mathrm{r}_{46} \mathrm{P}_{6 x}+2 \mathrm{P}_{4 \mathrm{x}} \mathrm{r}_{47} \mathrm{P}_{7 \mathrm{x}}+2 \mathrm{P}_{4 \mathrm{x}} \mathrm{r}_{48} \mathrm{P}_{8 \mathrm{x}}+2 \mathrm{P}_{4 \mathrm{x}} \mathrm{r}_{49} \mathrm{P}_{9 \mathrm{x}}+2 \mathrm{P}_{4 \mathrm{x}} \mathrm{r}_{10 \mathrm{x}}+2 \mathrm{P}_{5 \mathrm{x}} \mathrm{r}_{56} \mathrm{P}_{6 \mathrm{x}}+2 \mathrm{P}_{5 \mathrm{x}} \mathrm{r}_{57} \mathrm{P}_{7 \mathrm{x}}+2 \mathrm{P}_{5 \mathrm{x}} \mathrm{r}_{58} \mathrm{P}_{8 \mathrm{x}}+$ $2 \mathrm{P}_{5 \mathrm{x}} \mathrm{r}_{59} \mathrm{P}_{9 \mathrm{x}}+2 \mathrm{P}_{5 \mathrm{x}} \mathrm{r}_{510} \mathrm{P}_{10 \mathrm{x}}+2 \mathrm{P}_{6 \mathrm{x}} \mathrm{r}_{67} \mathrm{P}_{7 \mathrm{x}}+2 \mathrm{P}_{6 \mathrm{x}} \mathrm{r}_{68} \mathrm{P}_{8 \mathrm{x}}+2 \mathrm{P}_{6 \mathrm{x}} \mathrm{r}_{69} \mathrm{P}_{9 \mathrm{x}}+2 \mathrm{P}_{6 x} \mathrm{r}_{610} \mathrm{P}_{10 \mathrm{x}}+2 \mathrm{P}_{7 \mathrm{x}} \mathrm{r}_{78} \mathrm{P}_{8 \mathrm{x}}+2 \mathrm{P}_{7 \mathrm{x}} \mathrm{r}_{79} \mathrm{P}_{9 \mathrm{x}}+$ $2 \mathrm{P}_{7 \mathrm{x}} \mathrm{r}_{710} \mathrm{P}_{10 \mathrm{x}}+2 \mathrm{P}_{8 \mathrm{x}} \mathrm{r}_{89} \mathrm{P}_{9 \mathrm{x}}+2 \mathrm{P}_{810} \mathrm{P}_{10 \mathrm{x}}+2 \mathrm{P}_{9 \mathrm{x}} \mathrm{r}_{910} \mathrm{P}_{10 \mathrm{x}}$

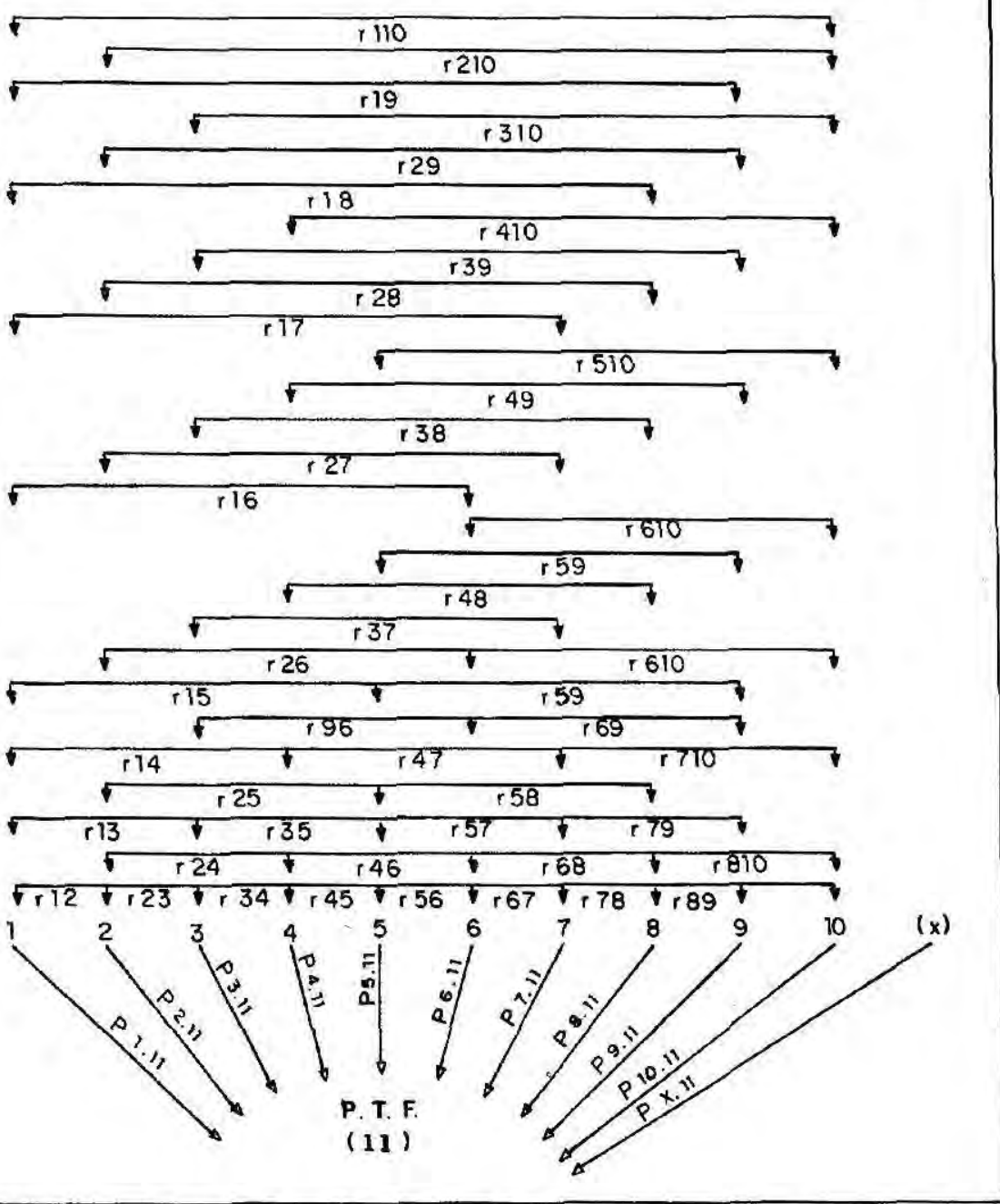

Figura 2. Diagrama da análise de trilha. Os $r$ 's representam os caracteres correlacionados em cubiu: DC(1), $\mathrm{AP}(2), \operatorname{AF}(3), \mathrm{LF}(4), \mathrm{CF}(5), \mathrm{NL}(6), \operatorname{EP}(7), \operatorname{NMF}(8), \operatorname{PMF}(9)$ e BRIX(10), Os P's são os coeficientes de caminhamento indicando a ação dos efeitos direto e indireto dos 10 caracteres sobre a PEF (11). $\mathrm{O} \mathbf{x}$ é a variável residual não correlacionada. 
RESULTADOS E DISCUSSÃO

Na Tabela 1, está discriminada a ação dos efeitos direto e indireto dos caracteres tratados como variáveis causais da produção estimada de frutos de cubiu. A análise de trilha mostrou que os caracteres DC, AP, CF, PMF e BRIX, apresentaram efeito direto negativo e os caracteres AF, LF, NL, EP e NMF efeito direto positivo sobre produção estimada de frutos de cubiu.

Com relação aos caracteres que manifestaram contribuição negativa para fins de seleção na produção de frutos de cubiu, observou-se que eles são muito influenciados indiretamente por outros caracteres de importância agronômica. Entretanto, aquele que exerceu influência mais marcante foi o NMF. Houve caso em que correlações negativas ocorreram entre NMF com LF, CF e PMF, e PMF entre EP e BRIX. Estes resultados são muito relevantes para outros pesquisadores que desejarem trabalhar no melhoramento do cubiu, porque evidenciam que será difícil, com essas populações no ambiente em que foram avaliadas, combinar adequadamente rendimento com outras caracteristicas de importância agronômicas. Isso quer dizer que uma intensidade de seleção maior em alguns deles, poderá resultar no afastamento de outros, com os quais se encontram correlacionados.

Outra observação importante que deve ser mencionada, é que, independentemente da espécie que se está trabalhando, percebe-se que o efeito direto do caráter altura da planta sobre a produção é sempre negativo. Esta constatação pode ser comprovada através dos resultados obtidos por RANGEL (1979) com arroz (Oriza sativa), OLIVEIRA et al. (1990) com feijão caupi (Vigna unguiculata) e PAIVA et al. (1982) com seringueira (Hevea brasiliensis). Esta predisposição da altura da planta afetar outras características pode ser explicada pela hipótese de FALCONER (1981). Ele admitiu que a interação do genótipo com o ambiente pode interferir no desenvolvimento de determinados caracteres de uma planta, por meio de diferentes mecanismos fisiológicos.

De qualquer forma, é aconselhável, de acordo com SHRIVASTAVA e SHARMA (1976), uma seleção cuidadosa das variáveis que se pretende estudar, e ao mesmo tempo, manter uma certa cautela quando utilizar determinada técnica. Estes pesquisadores numa pesquisa desenvolvida com arroz, avaliaram as variáveis número de perfilhos, número de grãos, peso de grãos e comprimento da panícula, através da análise de trilha. Os resultados encontrados mostraram que todas elas apresentaram efeito direto negativo sobre a produção de arroz. Com a eliminação da variável comprimento da panícula da análise, a contribuição das outras variáveis tornaram-se positivas. Se for tomado como base essa constatação, é possivel que a eliminação de algumas características, que não contribuiram positivamente direta ou indiretamente na produção de cubiu, pudessem favorecer o surgimento de mais características com efeitos positivos para fins de melhoramento da 
Tabela 1. Análise do coeficiente de caminhamento, desdobramento das correlações genotípicas em componentes de efeitos diretos e indiretos para os seguintes pares de caracteres: produção estimada de frutos $(\mathrm{PEF})$ vs diâmetro do caule, altura da planta $(\mathrm{AP})$, área da folha $(\mathrm{AF})$, número total médio de frutos por planta (NMF), largura do fruto (LF), comprimento do fruto (CF), peso médio dos frutos (PMF), espessura da polpa (EP), número de lóculos (NL) e teor de sólidos solúveis totais (BRIX).

\begin{tabular}{|c|c|c|c|}
\hline MODO DE AÇĀO & EFEITO DIRETO (P) & $\begin{array}{l}\text { EFEITO INDIRETO } \\
\text { (Pxr) }\end{array}$ & $\begin{array}{c}\text { CORRELAÇOES } \\
(\mathrm{r})\end{array}$ \\
\hline \multicolumn{4}{|l|}{ PEF vs DC } \\
\hline Efeito direto & $-0,047986$ & & \\
\hline Efeito indireto via AP & & $-0,051022$ & \\
\hline Efeito indireto via $\mathrm{AF}$ & & 0,114640 & \\
\hline Efeito indireto via NMF & & $-0,332464$ & \\
\hline Efeito indireto via LF & & 0,000161 & \\
\hline Efeito indireto via CF & & $-0,003763$ & \\
\hline Efeito indireto via PMF & & 0,000125 & \\
\hline Efeito indireto via EP & & 0,042477 & \\
\hline Efeito indireto via NL & & $-0,007593$ & \\
\hline Efeito indireto via BRIX & & 0,005433 & \\
\hline Total $\left(r_{p} \times D C\right)$ & & & $-0,71918$ \\
\hline \multicolumn{4}{|l|}{ PEF VS AP } \\
\hline Efeito direto & $-0,174136$ & & \\
\hline Efeito indireto via DC & & $-0,014060$ & \\
\hline Efeito indireto via AF & & 0,051062 & \\
\hline Efeito indireto via NMF & & 0,221221 & \\
\hline Efeito indireto via LF & & $-0,000553$ & \\
\hline Efeito indireto via CF & & 0,002696 & \\
\hline Efeito indireto via PMF & & 0,001072 & \\
\hline Efeito indireto via EP & & $-0,039850$ & \\
\hline Efeito indireto via NL & & $-0,016659$ & \\
\hline Efeito indireto via BRIX & & $-0,012254$ & \\
\hline Total $\left(r_{p} \times A P\right)$ & & & $-0,069784$ \\
\hline \multicolumn{4}{|l|}{ PEF vs AF } \\
\hline Efeito direto & 0,250306 & & \\
\hline Efeito indireto via DC & & $-0,021978$ & \\
\hline Efeito indireto via AP & & $-0,035524$ & \\
\hline Efeito indireto via NMF & & $-0,211108$ & \\
\hline Efeito indireto via LF & & 0,001032 & \\
\hline Efeito indireto via CF & & 0,004381 & \\
\hline Efeito indireto via PMF & & $-0,001145$ & \\
\hline Efeito indireto via EP & & $-0,039412$ & \\
\hline Efeito indireto via NL & & $-0,011446$ & \\
\hline Efeito indireto via BRIX & & $-0,004890$ & \\
\hline Total $\left(r_{p} \times A F\right)$ & & & $-0,069784$ \\
\hline \multicolumn{4}{|l|}{ PEF vs NMF } \\
\hline Efeito direto & 1,264120 & & \\
\hline Efeito indireto via DC & & 0,012620 & \\
\hline Efeito indireto via AP & & $-0,030474$ & \\
\hline Efeito indireto via AF & & $-0,041801$ & \\
\hline Efeito indireto via LF & & $-0,001435$ & \\
\hline Efeito indireto via CF & & 0,015616 & \\
\hline Efeito indireto via PMF & & 0,002456 & \\
\hline Efeito indireto via EP & & $-0,100719$ & \\
\hline Efeito indireto via NL & & $-0,020285$ & \\
\hline Efeito indireto via BRIX & & $-0,018230$ & \\
\hline Total ( $\left.r_{p} \times N M F\right)$ & & & 1,081868 \\
\hline
\end{tabular}


Cont. Tabela 1.

\begin{tabular}{|c|c|c|c|}
\hline MODODE AÇAO & EFEITO DIRETO (P) & $\begin{array}{c}\text { EFEITO INDIRETO } \\
\text { (Pxr) }\end{array}$ & $\begin{array}{c}\text { CORRELAÇOES } \\
(r)\end{array}$ \\
\hline \multicolumn{4}{|l|}{ PEF vs LF } \\
\hline Efeito direto & 0,005763 & & \\
\hline Efeito indireto via DC & & $-0,001344$ & \\
\hline Efeito indireto via AP & & 0,016717 & \\
\hline Efeito indireto via AF & & 0,044805 & \\
\hline Efeito indireto via NMF & & $-0,314766$ & \\
\hline Efeito indireto via CF & & $-0,017806$ & \\
\hline Efeito indireto via PMF & & $-0,009052$ & \\
\hline Efeito indireto via EP & & 0,290335 & \\
\hline Efeito indireto via NL & & 0,065048 & \\
\hline Efeito indireto via BRIX & & 0,006228 & \\
\hline Total $\left(r_{\rho} \times L F\right)$ & & & 0,085918 \\
\hline \multicolumn{4}{|l|}{ PEF vs CF } \\
\hline Efeito direto & $-0,056171$ & & \\
\hline Efeito indireto via DC & & 0,003215 & \\
\hline Efeito indireto via AP & & 0,008359 & \\
\hline Efeito indireto via AF & & $-0,019524$ & \\
\hline Efeito indireto via NMF & & $-0,315425$ & \\
\hline Efeito indireto via LF & & 0,001827 & \\
\hline Efeito indireto via PMF & & $-0,005838$ & \\
\hline Efeito indireto via EP & & 0,200563 & \\
\hline Efeito indireto via NL & & 0,016545 & \\
\hline Efeito indireto via BRIX & & 0,007787 & \\
\hline Total $\left(r_{p} \times C F\right)$ & & & $-0,201112$ \\
\hline \multicolumn{4}{|l|}{ PEF vs PMF } \\
\hline Efeito direto & $-0,010405$ & & \\
\hline Efeito indireto via DC & & 0,000576 & \\
\hline Efeito indireto via AP & & 0,017936 & \\
\hline Efeito indireto via AF & & 0,027534 & \\
\hline Efeito indireto via NMF & & $-0,298332$ & \\
\hline Efeito indireto via LF & & 0,005014 & \\
\hline Efeito indireto via CF & & $-0,031624$ & \\
\hline Efeito indireto via EP & & 0,307851 & \\
\hline Efeito indireto via NL & & 0,068902 & \\
\hline Efeito indireto via BRIX & & 0,004588 & \\
\hline Total $\left(r_{p} \times\right.$ PMF $)$ & & & 0,092040 \\
\hline \multicolumn{4}{|l|}{ PEF vs EP } \\
\hline Efeito direto & 0,437910 & & \\
\hline Efeito indireto via DC & & $-0,004656$ & \\
\hline Efeito indireto via AP & & 0,015846 & \\
\hline Efeito indireto via AF & & $-0,022528$ & \\
\hline Efeito indireto via NMF & & $-0,290748$ & \\
\hline Efeito indireto via LF & & 0,003821 & \\
\hline Efeito indireto via CF & & $-0,025726$ & \\
\hline Efeito indireto via PMF & & $-0,007315$ & \\
\hline Efeito indireto via NL & & 0,026971 & \\
\hline Efeito indireto via BRIX & & 0,013824 & \\
\hline Total $\left(r_{p} \times E P\right)$ & & & 0,147400 \\
\hline
\end{tabular}


Cont. Tabela 1.

\begin{tabular}{|c|c|c|c|}
\hline MODODE AÇAO & EFEITO DIRETO (P) & $\begin{array}{l}\text { EFEITO INDIRETO } \\
\text { (Pxr) }\end{array}$ & $\begin{array}{l}\text { CORRELAÇOES } \\
\text { (r) }\end{array}$ \\
\hline \multicolumn{4}{|l|}{ PEF vs NL } \\
\hline Eteito direto & 0,1113325 & & \\
\hline Efeito indireto via DC & & 0,003215 & \\
\hline Efeito indireto via AP & & 0,025598 & \\
\hline Eleito indireto via AF & & $-0,025281$ & \\
\hline Efeito indireto via NMF & & $-0,226278$ & \\
\hline Efeito indireto via LF & & 0,003308 & \\
\hline Efeito indireto via CF & & $-0,008201$ & \\
\hline Efeito indireto via PMF & & 0,006326 & \\
\hline Efeito indireto via EP & & 0,104223 & \\
\hline Efeito indireto via BRIX & & 0,007908 & \\
\hline Total $\left(r_{p} \times N L\right)$ & & & $-0,008509$ \\
\hline \multicolumn{4}{|l|}{ PEF vs BRIX } \\
\hline Eteito direto & $-0,060365$ & & \\
\hline Efeito indireto via DC & & 0,004319 & \\
\hline Efeito indireto via AP & & $-0,035350$ & \\
\hline Efeito indireto via AF & & 0,020275 & \\
\hline Efeito indireto via NMF & & 0,381764 & \\
\hline Efeito indireto via LF & & $-0,000594$ & \\
\hline Efeito indireto via CF & & 0,007146 & \\
\hline Efeito indireto via PMF & & 0,000791 & \\
\hline Efeito indireto via EP & & $-0,014846$ & \\
\hline Efeito indireto via NL & & 0,014687 & \\
\hline Total $\left(r_{0} \times B R I X\right)$ & & & 0.202059 \\
\hline
\end{tabular}

$\mathrm{R}^{2}(1,2,3, \ldots, 10){ }^{(1)} 0,725103$

$\mathrm{P}^{2}{ }_{\mathrm{x} 11}=1-\mathrm{R}^{2}{ }_{11}(1,2,3, \ldots, 10)^{(2)} 0,524306$

(1) Coeficiente de determinação

(2) Efeito da variável residual

produtividade dessa espécie.

Dos cinco caracteres que exerceram efeito direto e positivo sobre a produçâo de cubiu, todos são considerados de importância agronômica. $A$ área da folha (AF) tem um papel preponderante na taxa fotossintética da planta. A largura do fruto (LF) e o número de lóculos (NL) são características que estabelecem o padrão de uniformidade e firmeza dos frutos. A espessura da polpa (EP) indica o tipo de aproveitamento que o fruto terá na indústria $\mathrm{e}$, finalmente, o número total de frutos
(NMF) expressa todo o potencial da espécie em termos de produtividade. A análise de trilha identificou o caráter NMF como aquele que produziu maior efeito por via direta e indireta na produção de frutos. No confronto NMF vs PEF, observou-se que depois do desdobramento dos coeficientes de correlação em elementos de efeitos direto e indireto, o valor do coeficiente residual (Tabela 1), tornou-se maior que a unidade. Esta constatação é plenamente concebivel, porque está de acordo com as propriedades 
da análise de trilha definidas por WRIGHT (1934) e LI(1972 e 1975).

O grau de determinação genotipica da produção estimada de frutos de cubiu através das 10 variáveis consideradas (Tabela 1) assumiu o valor máximo: $\mathrm{R}^{2}{ }_{10}$ $(1,2,3, \ldots, 10)=0,725103$. Diante disto, pode-se concluir que $72,51 \%$ da variação da produção observada foi explicada geneticamente por estas variáveis.

$\mathrm{O}$ efeito da variável residual $\mathrm{P}_{11}^{2}=$ 0,524306 é considerado um valor aceitável. Isto quer dizer que alguns caracteres não se correlacionaram adequadamente entre si ou com a produção estimada de frutos (PEF). Na concepção de VASCONCELOS et al. (1983), um nivel de efeito da variável residual dessa magnitude pode ser plenamente concebível, desde que a ação dos fatores mensurados não tenham sido suficientes para mascarar a interpretação dos mesmos. Outra hipótese que também pode ser considerada para justificar a questão, é a própria natureza do germoplasma utilizado; além de ser representado por populações naturais pouco trabalhada agronomicamente, e estar sendo cultivado num ambiente muito diferente do seu centro de diversidade. De qualquer forma, ficou evidente que a análise de trilha forneceu um quadro diferente e mais completo da análise do coeficiente de correlação genética, indicando que a espessura da polpa e o número total de frutos são as características que podem promover progresso genético quando selecionadas para incrementar a produção de frutos de cubiu.

\section{CONCLUSÕES}

A análise de trilha sobre os coeficientes de correlação genética desdobrados demonstrou que:

1. Os caracteres diâmetro do caule (DC), altura da planta (AP), comprimento do fruto (CF), peso médio dos frutos (PMF) e teor de sólidos solúveis totais (BRIX) provocam efeito direto negativo na produção de frutos de cubiu.

2. Os caracteres área da folha (AF), largura da folha (LF), número de lóculos (NL), espessura da polpa (EP) e número total médio de frutos por planta (NMF) provocam efeito direto positivo na produção de cubiu.

3. Os caracteres espessura (EP) e número total médio de frutos por planta (NMF) podem promover progresso genético quando selecionados para incrementar a produção de frutos de cubiu.

4. O grau de determinação genotipica da produção total de cubiu através das 10 variáveis consideradas, demonstrou que $72,51 \%$ da variação da produção de frutos foi explicada genotipicamente por estas variáveis.

\section{Bibliografia citada}

BHATT, G.M. 1970. Multivariate analysis approach to selection of parents for hibridization aiming at yeld improvement in self-pollinated crops. Australian Joumal Agricutural Research, Victoria, 21:1-7.

CONSELHO DE DESENVOLVIMENTO DO ESTADO DE PERNAMBUCO (RECIFE,PE). 1987. Zoneamento pedoclimático do Estado de Pernambuco. Relatório de dados básicos. Recife: IPA SUDENE. 1, 183p.

CRUZ, C.D. 1990. Aplicação de algumas técnicas multivariadas no melhoramento de plantas. Piracicaba: ESALQ. 188p. Tese Doutorado.

DEWEI, D.R.; LU, K.H. 1959. A correlation and path-coefficient analysis of componentes of crested-wheatyrass seed production. Agronomy Journal, Madi- 
son, 51:515-519.

DUARTE, R.A.; ADAMS, M.W. 1972. A path coefficient analysis of some interrelation in field beans (Phaseolus vulgaris L.). Crop Science, Madison, 12:579-582.

ENCARNAÇẢO, C.R.F. 1980. Observaçōes meteorológicas e tipos climáticos das unidades e campos experimentais da Empresa Pernambucana de Pesquisa Agnopecuária. Recife: IPA, 100p.

FALCONER, D.S. 1981. Introduction to quantitative genetics. 2 ed. London: Longman, 340p.

INSTITUTO NACIONAL DE NUTRICION. 1977. Composicion de los alimentos peruanos.Lima: Ministério de Saludo, 329p.

LENKA, D; MISRA, B. 1973. Path coeflicient analysis of field rice varieties. Indian Journal of Agricultural Sciences, New Delhi, 43(4):367-369.

LI, C.C. 1972. Population genetics. London: The University of Chicago Press, 366p. 1975. Path analysis - a primer. Washington: Boxwood Press, 346p.

OLIVEIRA, F.J.; VAREJĀO-SILVA, M.A.; GOMES, M.J. 1990. Seleção de caracteres agronỏmicos de feijào (Phaseolus vulgaris $L$ ) usando coefientes de caminhamento, Pesquisa Brasileira, Brasilia, 25(7):1055-1064.

PAHLEN, A.V. D. 1977, Cubiu (Solanum topiro Humbl. \& Bonpl.), uma fruteira da Amazónia. Acta Amazonica. Manaus,
7:301-307.

PAIVA, J.R.; ROSSETI, A.G.; GONCALVES, P.S. 1982. Uso do coeficiente de caminhamento no melhoramento da seringueira. Pesquisa Agropecuaria Brasileira, Brasilia, 17(3):433-440.

RANGEL, P.H.N. 1979, Correlaçōes fenotipicas, genotipicas de ambiente e cocficientes de trilha, em variedades de arroz (Oriza sativa L.). Viçosa: UFV, 44p. Tese Mestrado.

SHRIVASTAVA, M.N.; SHARMA, K.K. 1976. Analysis of path coefficients in rice. Pflanzenzuchih, 77;174-177.

SILVA FILHO, D.F. 1994. Variabilidade genética em 29 populaçòes de cubiu (Solanum topiro Humbl. \& Bonpl. Solanacéae) avaliada na Zona da Mata do Estado de Pernambuco. Recife: UFRPE. $80 \mathrm{p}$. Tese Mestrado.

VASCONCELOS, M.E.C.; ABREU, C.P. 1992. Emprego do coeficiente de caminhamento em clones de seringueira. Pesquisa Agropecuaria Brasileira, Brasilia, 18(7):779-787, 1983.

VENCOVSKY, R.; BARRIGA, P. Associaçào entre caracteres. In: VENCOVSKY, R; BARRIGA, P. Genética biométrica no fitomelhoramento. Ribeirào Preto: Sociedade Brasileira de Genética, p. 33-486.

WRIGHT, S. 1934. The method of path coefficients. Annais of Mathematical Statistics, 5:161-215. 